\title{
Apremilast Prolongs the Time to First Biologic Therapy in Japanese Patients with Psoriasis
}

\author{
Masayuki Tanaka (D) - Yasushi Ozeki - Fujio Matsuyama • \\ Tatsunori Murata - Shinichi Imafuku - Taichi Nakamura
}

Received: October 11, 2021 / Accepted: November 27, 2021

(C) The Author(s) 2021, corrected publication 2022

\begin{abstract}
Introduction: Biologic agents are used in patients with severe psoriasis who have not adequately responded to existing conventional systemic therapies. However, only a limited number of medical institutions in Japan are approved to use them, and their relatively high cost represents a substantial burden to patients. Apremilast is an oral phosphodiesterase- 4 inhibitor approved in Japan for the treatment of psoriasis vulgaris in adult patients with an inadequate response to topical therapies and psoriatic arthritis in adult patients with active disease. To date, a large-scale real-world study of treatment patterns and costs associated with apremilast in Japan has not been conducted. The objective of this study was to assess whether
\end{abstract}

Supplementary Information The online version contains supplementary material available at https:// doi.org/10.1007/s13555-021-00659-w.

M. Tanaka $(\bowtie) \cdot$ Y. Ozeki · T. Nakamura

Inflammation and Immunology, General Medicine, Medical Affairs, Research and Development, Amgen

K.K., Midtown Tower 9-7-1 Akasaka, Minato-ku, Tokyo, Japan

e-mail: mtanak02@amgen.com

F. Matsuyama · T. Murata

Crecon Medical Assessment Inc., Tokyo, Japan

S. Imafuku

Fukuoka University, Fukuoka, Japan apremilast can prolong time to first biologic therapy use and decrease total medical cost.

Methods: Using the Medical Data Vision hospital-based claims database, 506 psoriasis patients were propensity score matched and analyzed (apremilast: $n=253$; non-apremilast: $n=253)$.

Results: The incidence rate of first biologic therapy use per 1000 patient-years was significantly lower in the apremilast group than in the non-apremilast group (30.3 vs. 107.6; $P<0.001)$, and the total medical costs per month were significantly lower in the apremilast group than in the non-apremilast group $(76,594$ yen/month vs. 102,411 yen/month, $P<0.001)$. In a sensitivity analysis of a propensity-score-matched subset of eligible patients prescribed biologics during the followup period (apremilast: $n=14$; non-apremilast: $n=14$ ), the incidence of first biologic therapy use was 2,797.6 per 1000 patient-years (95\% CI: $1,656.9,4,723.6)$ in the non-apremilast group and 856.1 per 1000 patient-years (95\% CI: 507.0, 1,445.5) in the apremilast group.

Conclusion: These results suggest that apremilast prolongs the time to first biologic therapy use in patients with psoriasis, thereby reducing the total medical cost and decreasing the economic burden on patients.

Keywords: Apremilast; Biologic therapy; Japan medical cost; Psoriasis 


\section{Key Summary Points}

Why carry out this study?

Biologic agents for the treatment of psoriasis may be associated with high clinical and economic burden among some patients who do not respond to topical therapies.

This study aimed to assess whether oral apremilast, approved for the treatment of psoriasis in Japan, could prolong the time to biologic use and reduce treatment costs.

\section{What was learned from the study?}

Patients treated with apremilast had a lower incidence of biologic use and longer time to biologic use compared with nonapremilast users.

Treatment with apremilast may ultimately reduce the clinical and cost burden on patients with psoriasis, demonstrating an unmet need for additional non-biologic therapies in this patient population.

\section{INTRODUCTION}

Treatments for psoriasis include topical agents, oral systemic agents, phototherapy, and biologic therapies. Treatment usually begins with topical corticosteroids or vitamin D3; if topical therapy is ineffective, phototherapy with ultraviolet A or B and systemic therapy with cyclosporine or oral etretinate (a vitamin A analog) are used. The Japanese guidance for the use of biologics for psoriasis specifies that biologics should be used in severe cases in which the patient does not sufficiently respond to existing systemic therapies [1].

Although biologics are highly efficacious, their use is currently limited to institutions approved by the Japanese Dermatological Association [1], where essential examinations 
database, was to calculate the time from the beginning of treatment to first biologic use in patients with psoriasis. Among patients who had an inadequate response to topical therapies, we analyzed the difference between two groups, namely those treated with apremilast and those not treated with apremilast. In addition, we evaluated the total medical costs for these treatment groups after they had initiated their first topical therapy, including treatment, testing, and hospitalization costs.

\section{METHODS}

\section{Study Database}

This retrospective cohort study analyzed healthcare claims from the Medical Data Vision database called EBM Provider. No institutional or ethics review was required because this analysis did not involve the collection, use, or transmittal of individually identifiable data.

EBM Provider is based on Diagnosis Procedure Combination (DPC) data and receipt data. Permission for secondary use of the data was obtained from DPC-utilizing hospitals. All data were anonymized to protect patients' personal information. DPC is a medical reimbursement system in Japan in which detailed data on, e.g., medical treatment, department, and procedures, are collected according to diagnosis. Data related to healthcare resource utilization (HCRU) outside of the individual medical institution are not captured, and the ability to track individual patients across participating hospitals is lacking. EBM Provider covers about 33 million patients at 419 institutions, which represents about $24 \%$ of DPC-utilizing hospitals in Japan [11]. Data from other dermatology clinics or non-DPC-utilizing hospitals were not captured.

\section{Study Period and Inclusion/Exclusion Criteria}

The study period was from January 2015 to June 2019 (the latest date of available data when the analysis was performed). In the analyses of time to first biologic therapy use and medical costs, the earliest date of a psoriasis medication prescription that fell after a diagnosis code for psoriasis vulgaris (International Classification of Diseases, Tenth Edition, code L40.0 or L40.9) or psoriatic arthritis (International Classification of Diseases, Tenth Edition, code L40.5) was set as the index date. The period from the index date to the last day of the month, corresponding to the last day that information was recorded in EBM Provider, was set as the follow-up period (Fig. 1). In the analyses of time to first biologic therapy use, only biologics indicated for psoriasis vulgaris or psoriatic arthritis in Japan were included (i.e., adalimumab, infliximab, secukinumab, ixekizumab, ustekinumab, brodalumab, and guselkumab). Patients with psoriasis who started treatment with a topical therapy only and received systemic treatment after topical therapy were included if they met the following inclusion criteria: (1) had two or more diagnosis codes for psoriasis vulgaris or psoriatic arthritis; (2) had been prescribed a therapeutic drug for psoriasis after diagnosis codes for psoriasis vulgaris or psoriatic arthritis; (3) had been prescribed a topical skin preparation for psoriasis at the index date; (4) had been prescribed an oral (apremilast, etretinate, epinastine, olopatadine, oral corticosteroids, cyclosporine, or methotrexate) or biologic therapy for psoriasis after the index date; (5) had not been prescribed a therapeutic drug for psoriasis at the baseline period (180 days before the index date); and (6) were $\geq 18$ years of age at the index date (Figs. 1, 2).

Patients were excluded from the analysis if they: (1) had any diagnosis codes for psoriasis other than psoriasis vulgaris or psoriatic arthritis; (2) did not have a 180-day baseline period; (3) had a follow-up period of $<1$ month; or (4) were prescribed oral or biologic therapy for psoriasis at the index date (Figs. 1, 2).

\section{Cohort Definition}

Patients who met the eligibility criteria and were prescribed apremilast during the follow-up period were defined as the apremilast group, and those who were not prescribed apremilast 
Study Period: Jan/2015-Jun/2019

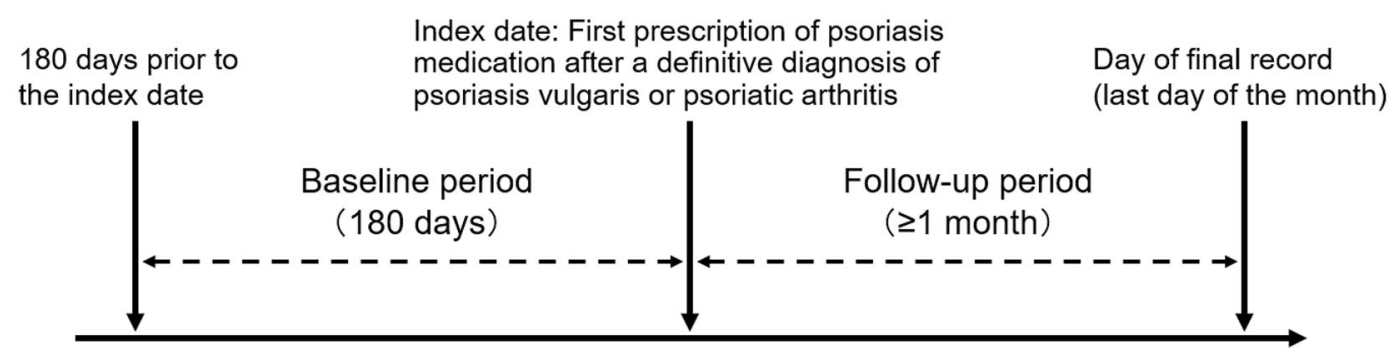

Fig. 1 Definition of the index date, baseline period, and follow-up period

during the follow-up period were defined as the non-apremilast group.

\section{Statistical Analysis}

To improve comparability, 1:1 propensity score matching using the nearest-neighbor method was used to compare cohorts and reduce the risk of confounding. A logistic regression analysis was conducted, adjusting for age at the index date, sex, disease type (psoriasis vulgaris/psoriatic arthritis), presence or absence of comorbidities (obesity, diabetes mellitus, hypertension, dyslipidemia), frequency of hospitalization during the baseline period, cumulative days of hospitalization during the baseline period, cumulative number of outpatient visits during the baseline period, duration of follow-up, starting dose of topical corticosteroids, mean dose of topical corticosteroids until oral or biologic agents were prescribed, starting dose of topical nonsteroidal agents, and mean dose of topical nonsteroidal agents until oral or biologic agents were prescribed. The patient demographics for the apremilast and non-apremilast groups were compared for the pre-matching and post-matching groups, and a balance check was performed using the standardized mean difference (SMD).

For the analysis of time to first biologic therapy use, the incidence per 1000 personyears of the first introduction of a biologic during the follow-up period after the index date and its 95\% confidence interval (CI) were calculated. The chi-square test was used to compare the percentage of biologic therapy use between treatment groups. Kaplan-Meier estimates of the cumulative incidence of the first use of a biologic during the follow-up period were calculated, and the times to the 25 th percentile, 50th percentile, and 75th percentile of the cumulative incidence were calculated. A log-rank test was used for between-group comparisons.

To determine monthly HCRU, the following formula was used:

HCRU $=\frac{\text { HCRU during follow-up period (yen) }}{\text { Follow-up period (number of months) }}$

In addition, the monthly cost after the index date was calculated for each patient, and a figure showing the time course of the mean cost per month by group plotted against the number of months from the index date on the horizontal axis was generated.

For continuous data, the mean, standard deviation, minimum, median, and maximum values for the number of eligible patients were reported. For categorical data, the number of applicable subjects and its proportion of the total were reported.

All analyses were performed using SAS ver. 9.4 (SAS Institute Inc., Cary, NC, USA). A sensitivity analysis using an inverse probability of treatment weighting (IPTW) method was also performed for a subset of eligible patients from both treatment groups who were prescribed biologics during the follow-up period (see Supplemental Methods). 


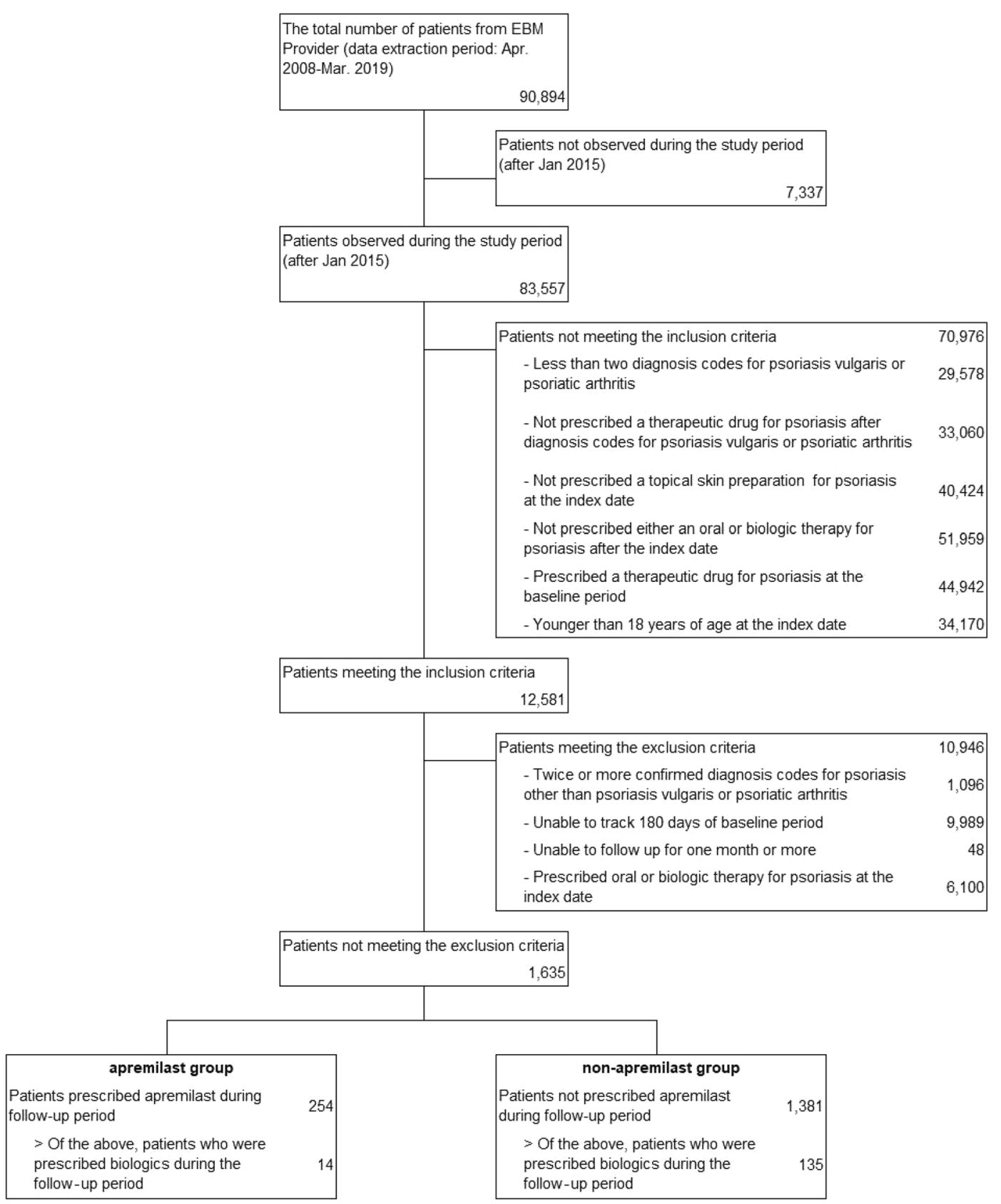

Fig. 2 Patient attrition

\section{RESULTS}

\section{Study Population}

A total of 83,557 patients had a diagnosis of psoriasis during the study period, and 1635 patients (apremilast: $n=254$; non-apremilast: $n=1381)$ met the eligibility criteria during the study period (Fig. 2).

\section{Patient Characteristics}

The number of patients in each group after propensity score matching and the patient 
Table 1 Patient characteristics after propensity score matching

\begin{tabular}{|c|c|c|c|c|}
\hline & \multicolumn{4}{|c|}{ After propensity score matching } \\
\hline & $\begin{array}{l}\text { Apremilast group } \\
n=253\end{array}$ & $\begin{array}{l}\text { Non-apremilast group } \\
n=253\end{array}$ & $\begin{array}{l}\text { Total } \\
n=506\end{array}$ & SMD $^{\mathrm{a}}$ \\
\hline Age, mean (SD), years, mean (SD) & $63.1(16.0)$ & $62.1(14.6)$ & $62.6(15.3)$ & \\
\hline \multicolumn{5}{|l|}{ Age category, $n(\%)$} \\
\hline$<50$ & $53(20.9)$ & $55(21.7)$ & $108(21.3)$ & \\
\hline $50-59$ & $40(15.8)$ & $46(18.2)$ & $86(17.0)$ & \\
\hline $60-69$ & $55(21.7)$ & $68(26.9)$ & $123(24.3)$ & \\
\hline$\geq 70$ & $105(41.5)$ & $84(33.2)$ & $189(37.4)$ & \\
\hline Male, $n(\%)$ & $154(60.9)$ & $154(60.9)$ & $308(60.9)$ & 0.00 \\
\hline \multicolumn{5}{|l|}{ Disease type, $n(\%)$} \\
\hline Psoriasis vulgaris only & $240(94.9)$ & $242(95.7)$ & $482(95.3)$ & 0.04 \\
\hline $\begin{array}{l}\text { Comorbid psoriasis vulgaris and psoriatic } \\
\text { arthritis }\end{array}$ & $9(3.6)$ & $6(2.4)$ & $15(3.0)$ & -0.07 \\
\hline Psoriatic arthritis only ${ }^{\mathrm{b}}$ & $4(1.6)$ & $5(2.0)$ & $9(1.8)$ & 0.03 \\
\hline \multicolumn{5}{|l|}{ Diagnosis, $n(\%)$} \\
\hline Obesity & $3(1.2)$ & $1(0.4)$ & $4(0.8)$ & -0.09 \\
\hline Diabetes & $50(19.8)$ & $37(14.6)$ & $87(17.2)$ & -0.12 \\
\hline Hypertension & $53(20.9)$ & $30(11.9)$ & $83(16.4)$ & -0.20 \\
\hline Dyslipidemia & $48(19.0)$ & $39(15.4)$ & $87(17.2)$ & -0.09 \\
\hline \multicolumn{5}{|c|}{ Treatment information during the baseline period, mean (SD) } \\
\hline Hospitalization frequency & $0.12(0.40)$ & $0.07(0.29)$ & $0.09(0.35)$ & 0.10 \\
\hline Length of hospital stay, days & $0.9(4.2)$ & $0.7(4.4)$ & $0.8(4.3)$ & 0.03 \\
\hline Number of patients visits & $3.4(6.0)$ & $2.8(5.8)$ & $3.1(5.9)$ & 0.07 \\
\hline Direct medical cost, yen & $182,435(742,129)$ & $132,292(362,603)$ & $\begin{array}{l}157,363 \\
\quad(584,014)\end{array}$ & 0.06 \\
\hline Length of follow-up period, months & $20.8(12.5)$ & $20.9(14.1)$ & $20.8(13.3)$ & 0.00 \\
\hline $\begin{array}{l}\text { Period until oral drug or biologic for psoriasis is } \\
\text { prescribed, days }\end{array}$ & $289.7(309.9)$ & $199.7(240.3)$ & $249.2(284.0)$ & 0.29 \\
\hline \multicolumn{5}{|l|}{ Use of psoriasis drug during the follow-up period } \\
\hline Use of steroidal topical agent, $n(\%)$ & $249(98.4)$ & $249(98.4)$ & $498(98.4)$ & 0.00 \\
\hline Use of non-steroidal topical agent, $n$ (\%) & $244(96.4)$ & $213(84.2)$ & $457(90.3)$ & -0.33 \\
\hline $\begin{array}{l}\text { Initial dose of steroidal topical agent, mean } \\
(\mathrm{SD}), \mathrm{g}^{\mathrm{c}}\end{array}$ & $61.2(51.6)$ & $61.4(51.3)$ & $61.3(51.4)$ & 0.00 \\
\hline
\end{tabular}


Table 1 continued

\begin{tabular}{|c|c|c|c|c|}
\hline & \multicolumn{4}{|c|}{ After propensity score matching } \\
\hline & $\begin{array}{l}\text { Apremilast group } \\
n=253\end{array}$ & $\begin{array}{l}\text { Non-apremilast group } \\
n=253\end{array}$ & $\begin{array}{l}\text { Total } \\
n=506\end{array}$ & SMD $^{a}$ \\
\hline $\begin{array}{l}\text { Maximum dose of steroidal topical agent, mean } \\
(\mathrm{SD}), \mathrm{g}^{\mathrm{c}}\end{array}$ & $142.2(125.3)$ & $106.8(98.2)$ & $124.5(113.8)$ & 0.30 \\
\hline $\begin{array}{l}\text { Average dose of steroidal topical agent, mean } \\
(\mathrm{SD}), \mathrm{g}^{\mathrm{c}}\end{array}$ & $2.61(3.28)$ & $2.15(3.22)$ & $2.38(3.25)$ & 0.09 \\
\hline \multicolumn{5}{|l|}{ Class of steroidal topical agent, initial dose ${ }^{c}, n(\%)$} \\
\hline Strongest & $45(17.8)$ & $39(15.4)$ & $84(16.6)$ & \\
\hline Very strong & $193(76.3)$ & $178(70.4)$ & $371(73.3)$ & \\
\hline Strong & $6(2.4)$ & $24(9.5)$ & $30(5.9)$ & \\
\hline Medium & $5(2.0)$ & $8(3.2)$ & $13(2.6)$ & \\
\hline Weak & $0(0.0)$ & $0(0.0)$ & $0(0.0)$ & \\
\hline Missing & $4(1.6)$ & $4(1.6)$ & $8(1.6)$ & \\
\hline \multicolumn{5}{|l|}{ Class of steroidal topical agent, strongest class ${ }^{\mathrm{c}}, n$ (\%) } \\
\hline Strongest & $105(41.5)$ & $83(32.8)$ & $188(37.2)$ & \\
\hline Very strong & $141(55.7)$ & $145(57.3)$ & $286(56.5)$ & \\
\hline Strong & $1(0.4)$ & $20(7.9)$ & $21(4.2)$ & \\
\hline Medium & $2(0.8)$ & $1(0.4)$ & $3(0.6)$ & \\
\hline Weak & $0(0.0)$ & $0(0.0)$ & $0(0.0)$ & \\
\hline Missing & $4(1.6)$ & $4(1.6)$ & $8(1.6)$ & \\
\hline \multicolumn{5}{|c|}{ Class of steroidal topical agent, most frequently used class ${ }^{\mathrm{c}}, n(\%)$} \\
\hline Strongest & $34(13.4)$ & $30(11.9)$ & $64(12.6)$ & \\
\hline Very strong & $197(77.9)$ & $175(69.2)$ & $372(73.5)$ & \\
\hline Strong & $9(3.6)$ & $25(9.9)$ & $34(6.7)$ & \\
\hline Medium & $5(2.0)$ & $6(2.4)$ & $11(2.2)$ & \\
\hline Weak & $0(0.0)$ & $0(0.0)$ & $0(0.0)$ & \\
\hline Missing & $8(3.2)$ & & $25(4.9)$ & \\
\hline $\begin{array}{l}\text { Initial dose of non-steroidal topical agent, mean } \\
\text { (SD), g }\end{array}$ & $55.7(44.8)$ & $50.5(44.3)$ & $53.1(44.6)$ & 0.12 \\
\hline $\begin{array}{l}\text { Maximum dose of non-steroidal topical agent, } \\
\text { mean (SD), g }\end{array}$ & $109.1(90.2)$ & $85.3(93.3)$ & $97.2(92.5)$ & 0.24 \\
\hline $\begin{array}{l}\text { Average dose of non-steroidal topical agent, } \\
\text { mean (SD), g }\end{array}$ & $1.96(2.53)$ & $1.68(2.76)$ & $1.82(2.65)$ & 0.11 \\
\hline
\end{tabular}


Table 1 continued

\begin{tabular}{llllr}
\hline & \multicolumn{3}{l}{ After propensity score matching } & \\
\cline { 2 - 5 } & $\begin{array}{l}\text { Apremilast group } \\
\boldsymbol{n}=\mathbf{2 5 3}\end{array}$ & $\begin{array}{l}\text { Non-apremilast group } \\
\boldsymbol{n}=\mathbf{2 5 3}\end{array}$ & $\begin{array}{l}\text { Total } \\
\boldsymbol{n}=\mathbf{5 0 6}\end{array}$ & SMD $^{\mathbf{a}}$ \\
\hline Total prescription of topical agent, mean (SD), g & $1318.1(1780.7)$ & $720.6(1069.8)$ & $\begin{array}{c}1019.3 \\
(1497.6)\end{array}$ & 0.42 \\
Prescription of topical agent per day, mean (SD), & $2.30(2.64)$ & $1.49(1.98)$ & $1.90(2.36)$ & 0.36 \\
$\quad$ g & $35(13.8)$ & $34(13.4)$ & $69(13.6)$ & -0.01 \\
Use of oral etretinate, $n(\%)$ & $8(3.2)$ & $17(6.7)$ & $25(4.9)$ & 0.18 \\
Use of oral cyclosporine, $n(\%)$ & $13(5.1)$ & $40(15.8)$ & $53(10.5)$ & 0.40 \\
Use of biologic, $n(\%)$ & & &
\end{tabular}

$S D$ standard deviation; $S M D$ standardized mean difference

${ }^{a}$ Non-apremilast group as reference

${ }^{b}$ Patients who had a code for psoriatic arthritis only were considered to also have psoriasis vulgaris if they had prescription records for topical therapies at the index date

${ }^{\mathrm{c}}$ Aggregated period for initial dose, maximum dose, class at the start of use, and the strongest class: follow-up period; aggregated period for average dose and the most frequently used class: until oral drugs or biologics for psoriasis were presented during the follow-up period

demographics are shown in Table 1 . The postmatching population consisted of 253 patients per group with a mean age of approximately 63 years, of whom approximately 60\% were men. Approximately $95 \%$ of the patients had psoriasis vulgaris only, $2 \%$ had psoriatic arthritis only, and the remaining 3\% had comorbid psoriasis vulgaris and psoriatic arthritis.

Although the mean follow-up period was approximately 21 months (630 days) in both treatment groups, the mean time to first oral or biologic therapy use was 199.7 days in the nonapremilast group and 289.7 days in the apremilast group. During the follow-up period, no significant difference was observed in the mean dose of topical corticosteroids until oral or biologic therapies were prescribed between the two treatment groups; however, there was a tendency toward higher use of topical steroids in the apremilast group. No difference was noted in the presence or absence of an etretinate prescription during the follow-up period between the two treatment groups $(\mathrm{SMD}=-0.01)$, but the number of cyclosporines and biologics prescribed was higher in the non-apremilast group
$(\mathrm{SMD}=0.18$ for cyclosporines and 0.40 for biologics) (Table 1).

SMDs of items not included as covariates in the logistic regression analysis for calculating the propensity score (i.e., duration from the start of follow-up to the prescription of oral or biologic therapies for psoriasis, total amount of topical skin preparation prescribed during the follow-up period, amount of topical skin preparation prescribed per day, and presence or absence of a biologic prescription) were relatively large. The values of other parameters generally fell within or close to the range of -0.1 to 0.1 , showing no significant difference between the treatment groups (Fig. 3). The patient characteristics before propensity score matching are shown in Supplemental Table S1.

\section{Time to First Biologic Therapy Use}

The incidence rate of first biologic therapy use during the follow-up period was 30.3 per 1000 person-years $(95 \%$ CI: 17.6, 52.2) in the apremilast group and 107.6 per 1000 personyears $(95 \% \mathrm{CI}: 78.9,146.7)$ in the non-apremilast-group (Table 2). 


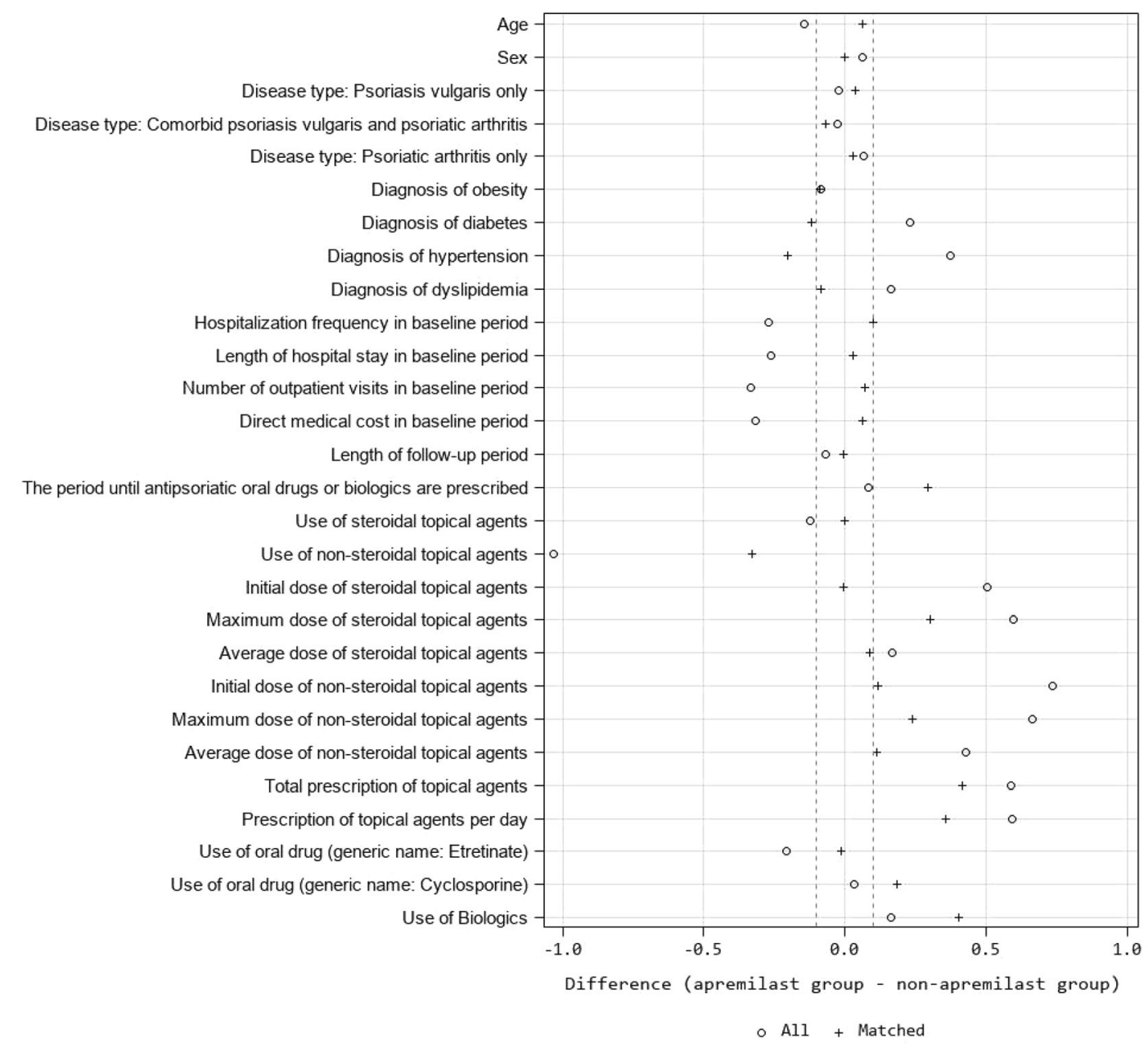

Fig. 3 Standardized mean differences plot

Table 2 Incidence of biologic therapy

$n \quad$ Biologic therapy incidence rate (per 1000 person-years)

\begin{tabular}{lllllll} 
& & Biologic therapy & Person-years & Incidence rate & $\mathbf{9 5 \%} \mathbf{C I}^{\mathbf{a}}$ & $\boldsymbol{P}_{\text {value }}$ \\
\hline Apremilast group & 253 & 13 & 429.2 & 30.3 & $17.6,52.2$ & \\
Non-apremilast group & 253 & 40 & 371.7 & 107.6 & $78.9,146.7$ & $<0.001$ \\
\hline
\end{tabular}

$C I$ confidence interval

${ }^{\mathrm{a}}$ 95\% CI was calculated using the Poisson distribution

${ }^{\mathrm{b}} \mathrm{Chi}$-square test

The cumulative incidence of first biologic therapy use during the follow-up period was estimated using the Kaplan-Meier method and was significantly lower in the apremilast group $(P<0.001)$ (Fig. 4).

\section{HCRU}

The results for each HCRU endpoint are shown in Table 3. The frequency of outpatient visits per month was significantly higher in the 


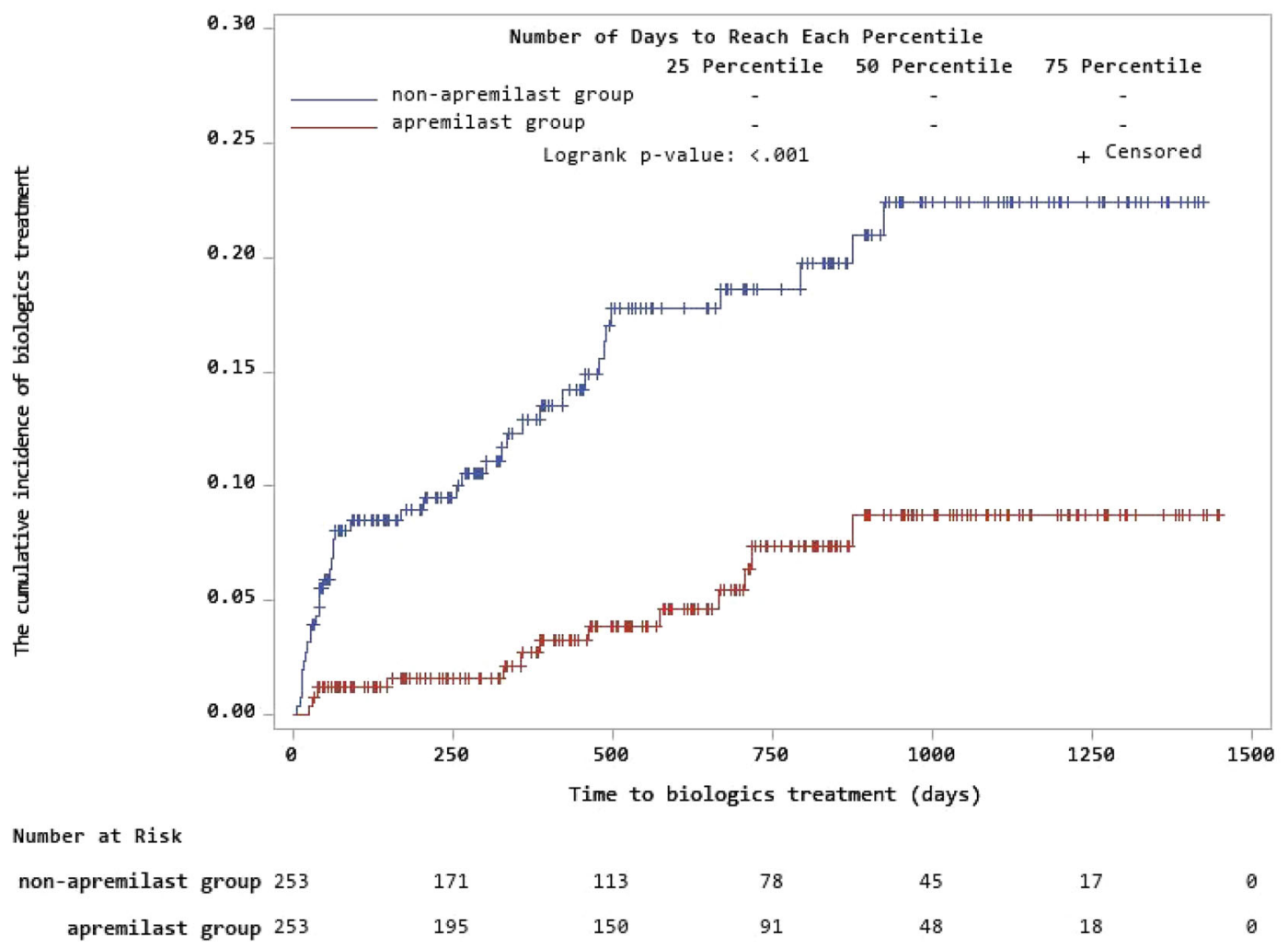

Fig. 4 Cumulative incidence of biologic therapy

apremilast group compared with the nonapremilast group (1.54 vs. $1.17 ; P<0.001)$. The apremilast group also had significantly greater per-month costs for topical agents $(8,830$ yen vs. 4,910 yen; $P<0.001$ ), phototherapy (761 yen vs. 215 yen; $P<0.001$ ), and oral therapies other than methotrexate $(19,285$ yen vs. 1,458 yen; $P<0.001)$ than the non-apremilast group. On the other hand, the apremilast group had a significantly lower cost per month for biologics $(4,887$ yen vs. 26,481 yen; $P<0.001)$ or methotrexate (42 yen vs. 77 yen; $P=0.044$ ) than the non-apremilast group and also had a significantly lower total medical cost per month than the non-apremilast group (mean [median, minimum-maximum], respectively: 76,594 yen [52,444 yen, 4,467-1,234,008 yen] in the apremilast group vs. 102,411 yen $[26,849$ yen, $1,455-2,484,115$ yen] in the non-apremilast group; $P<0.001$ ).

The results for the monthly changes in each HCRU cost item after the index date are presented as supplemental data (Fig. S1A-K). The apremilast group had higher mean topical skin agent, phototherapy, and oral therapy (other than methotrexate) costs for psoriasis throughout the follow-up period, although it could not be inferred from the database whether these therapies had been used before, after, or concomitantly with apremilast. On the other hand, the non-apremilast group had higher mean methotrexate and biologic costs for psoriasis in the latter half of the follow-up period. Although the total medical costs did not differ between treatment groups immediately after the start of follow-up, they tended to increase in the non-apremilast group over time as the cost of biologic therapies increased.

\section{Sensitivity Analysis}

Of the eligible patients, 149 (apremilast: $n=14$; non-apremilast: $n=135$ ) were prescribed biologics during the follow-up period. When the 
Table 3 Healthcare resource utilization

\begin{tabular}{|c|c|c|c|c|}
\hline & $\begin{array}{l}\text { Apremilast group, mean } \\
\text { (SD) } n=253\end{array}$ & $\begin{array}{l}\text { Non-apremilast group, mean } \\
\text { (SD) } n=253\end{array}$ & $\begin{array}{l}\text { Absolute } \\
\text { difference }\end{array}$ & $P$ value $^{\mathrm{a}}$ \\
\hline $\begin{array}{l}\text { Number of outpatient } \\
\text { visits/month }\end{array}$ & $1.54(1.05)$ & $1.17(1.06)$ & 0.36 & $<0.001$ \\
\hline \multicolumn{5}{|l|}{ Direct medical cost } \\
\hline \multicolumn{5}{|l|}{$\begin{array}{l}\text { Cost of psoriasis drugs/ } \\
\text { treatment procedure }\end{array}$} \\
\hline Topical agents, yen/month & $8830(9259)$ & $4910(6335)$ & 3920 & $<0.001$ \\
\hline Phototherapy, yen/month & $761(1924)$ & $215(1077)$ & 546 & $<0.001$ \\
\hline $\begin{array}{l}\text { Oral drugs (except } \\
\text { methotrexate), yen/month }\end{array}$ & $19,285(17,391)$ & $1458(3993)$ & 17,827 & $<0.001$ \\
\hline Methotrexate, yen/month & $42(411)$ & $77(507)$ & -34 & 0.044 \\
\hline Biologic agents, yen/month & $4887(26,013)$ & $26,481(74,478)$ & $-21,594$ & $<0.001$ \\
\hline $\begin{array}{l}\text { Other drugs for psoriasis, } \\
\text { yen/month }\end{array}$ & $2(9)$ & $20(205)$ & -19 & 0.138 \\
\hline Cost of other drugs, yen/month & $16,035(82,181)$ & $20,104(57,021)$ & -4070 & 0.378 \\
\hline $\begin{array}{l}\text { Cost of other treatment } \\
\text { procedure, yen/month }\end{array}$ & $3648(22,619)$ & $8842(72,078)$ & -5195 & 0.624 \\
\hline Cost of tests, yen/month & $4425(5254)$ & $7201(17,990)$ & -2776 & 0.162 \\
\hline $\begin{array}{l}\text { Basic cost of hospitalization, } \\
\text { yen/month }\end{array}$ & $8924(24,893)$ & $19,845(78,327)$ & $-10,921$ & 0.471 \\
\hline Total cost, yen/month & $76,594(107,738)$ & $102,411(214,261)$ & $-25,817$ & $<0.001$ \\
\hline
\end{tabular}

$S D$ standard deviation

${ }^{a}$ Wilcoxon rank-sum test

incidence rate of first biologic therapy use was calculated for 14 patients in each treatment group matched by propensity score, the incidence was 856.1 per 1000 patient-years (95\% CI: $507.0,1,445.5)$ in the apremilast group and 2,797.6 per 1000 patient-years (95\% CI: 1,656.9, $4,723.6$ ) in the non-apremilast group (Supplemental Table S2). In addition, the cumulative incidence of first biologic therapy use estimated using the Kaplan-Meier method was significantly lower in the apremilast group $(P<0.001)$ (Supplemental Fig. S2). Thus, the incidence rate was lower in the apremilast group than in the non-apremilast group, comparable to the results for the entire population of eligible patients (Table 2).

The results of sensitivity analysis using the IPTW method are reported as supplemental data (Table S3, S4, Fig. S3). As seen in the main analysis, a decrease in the incidence rate of first biologic therapy use, a prolongation of the time to first biologic therapy use, and a reduction in medical costs were confirmed for apremilast.

\section{DISCUSSION}

This study showed a significantly lower incidence rate of first biologic therapy use in psoriasis patients taking apremilast vs. comparable 
psoriasis patients not taking apremilast using a Japanese claims database. To our knowledge, this is the first large-scale, nationwide, realworld data study to look at apremilast treatment patterns and costs in Japan.

Biologics for psoriasis are highly efficacious; however, several biologic-associated burdens are suggested by the literature and clinical practice. For example, in a discrete choice experiment study conducted by Tada et al. in Japanese patients with psoriasis to investigate preferences for biologics that differ in administration frequency, price, efficacy, and safety, it was reported that patients preferred treatments with a lower injection frequency and a lower cost burden [12]. In addition, biologics can only be used at a limited number of medical institutions, which may entail transferring patients to approved hospitals for treatment. Thus, apremilast may have the potential to reduce the treatment burden associated with biologics by decreasing the use of or prolonging the time to first biologic therapy use. The favorable safety profile, oral mode of administration (injection is used for many biologics), and lower cost of apremilast may also contribute to patient treatment choice. Finally, differences in treatment choices and time to initiation of biologic therapy may be influenced by the patient's therapeutic goals, disease severity, quality-oflife impacts, and success with previous treatments.

Although apremilast may decrease the burden of biologic therapy, the apremilast group had a higher number of outpatient visits per month than the non-apremilast group (1.54 vs $1.17, P<0.001$; Table 3). Although the number of outpatient visits was calculated during whole follow-up periods after the index date (including visits before apremilast or biologic prescription), high frequencies of outpatient visits may be a burden of apremilast treatment. On the other hand, in Japan, prescription amounts for new medications are limited by regulation to 2 weeks at a time until 1 year after their launch, which could have resulted in high frequencies of outpatient visits for some of the apremilasttreated patients in this study.

When this research was conducted, the latest date of available data was June 2019; the duration of the research period after the launch of apremilast was at most about 2 years (from March 2017 to June 2019). The results might have been different if the duration of the research period was longer. In the analysis of the monthly change in each HCRU cost item after the index date, the monthly cost of biologic therapies tended to increase greatly in the latter half of the follow-up period in the nonapremilast group, but was lower and relatively stable in the apremilast group throughout the entire follow-up period (Supplemental Fig. S1E). Therefore, this analysis using data from until about 2 years after apremilast launch may underestimate the costs of biologics; a greater reduction in medical costs for apremilast could potentially be observed if a longer analysis was performed. Among eligible patients, the incidence of biologic prescriptions and the time to first biologic therapy use were also analyzed for patients who were prescribed biologics during the follow-up period, but the CIs were wide and the point estimates unstable due to the small sample size. These points should be re-evaluated when more data are accumulated in the future.

Regarding the burden of medical costs, our study showed that the mean total medical cost per month after the start of treatment for psoriasis was significantly lower in patients taking apremilast than in those not taking apremilast. This finding is consistent with three US studies that used real-world data to compare healthcare costs for patients initiating treatment with apremilast or biologics [13-15]. On the other hand, it should be noted that the costs in this study were calculated during whole follow-up periods after the index date; the costs included those before the initiation of apremilast. Although the costs for the period after apremilast initiation could not be analyzed due to the limitations of the study design, this should be further investigated by selecting appropriate comparison groups in future studies.

Prescription costs can be a barrier to biologic use, and the treatment cost of biologic therapies in Japan has been reported to be about 1.2 million yen to 4.4 million yen per year [16]. A study by Sruamsiri et al. [17] compared medical expenses for 12 months before and after the start of biologics using Japanese claims data, 
and reported that the start of biologics increased medical expenses by about 1.9 million yen per patient for the first year after biologic initiation, of which about 1.8 million yen was accounted for by outpatient expenses. In the current study, the cost item with the largest between-group difference was the average cost of biologic agents (the absolute difference between the apremilast and non-apremilast groups was 21,594 yen), and the major contributor to the high average total medical cost in the non-apremilast group was the high prices of biologics.

On the other hand, in our study, the average cost per month of topical agents, phototherapy, and oral drugs except for methotrexate (apremilast may have been a major contributor to this cost) was significantly higher in the apremilast group (Table 3). Due to the limitations of the study design, the cost includes the whole follow-up period (i.e., before, during, and after the apremilast prescription). In terms of topical agents, the use of apremilast in combination with topical therapy was effective in patients who were dissatisfied with stable maintenance topical therapy [18]. In addition, the effectiveness of the combination of apremilast and narrowband ultraviolet $\mathrm{B}$ has been reported in patients with moderate to severe psoriasis [19]. In accordance with the literature, the results of this study raise the possibility that apremilast may be preferentially used with topical therapy or phototherapy in clinical settings. A future real-world data study designed to evaluate the use of monotherapy and combination therapy with apremilast is needed.

\section{Study Limitations}

This study has several limitations.

First, the study used claims data from the EBM Provider database. Information on treatment selection is not included in the EBM Provider database. Therefore, it is unclear as to why patients were or were not initiated on apremilast. In addition, results from this study show that apremilast may reduce the treatment burden associated with biologics, and data on drug efficacy are not included in the EBM Provider database, so efficacy could not be compared with the apremilast-naive group. Similarly, further research is needed to evaluate the impact of other available oral therapies on the treatment burden associated with biologics. In addition, because information on the severity of psoriasis could not be obtained, and matching the two groups in terms of refractory patients with topical therapy was not possible, the possibility that time to first biologic therapy use was prolonged because more patients had less severe psoriasis in the apremilast group cannot be excluded. However, since similar results were obtained when the analysis was performed only in patients who were prescribed biologics during the follow-up period (Supplemental Fig. S2), the prolongation of time to biologic use in the apremilast group was unlikely to be caused only by a difference in the proportion of refractory patients with topical therapy or by differences in severity between treatment groups. Lastly, it is also possible that, because biologics can only be administered at a limited number of medical institutions in Japan, patients may have visited these DPC-utilizing hospitals for the sake of starting biologics.

The second point is attributed to the study design. In this study, the apremilast group and the non-apremilast group were matched using propensity scores. Because the number of patients in the non-apremilast group was larger, and $>80 \%$ of the eligible patients were excluded after matching, the matched non-apremilast group may not represent the entire nonapremilast cohort. Regarding this point, similar results were obtained in the main analysis when sensitivity analyses were performed by the IPTW method. Therefore, it is likely that the results of this study are relatively robust. The reason that the incidence of biologic prescriptions in the non-apremilast group was lower when the IPTW method was used than when the matching method was used may be that the IPTW method estimates the average treatment effect in the entire psoriasis patient population and thus includes patients with relatively mild psoriasis who were not eligible for treatment with apremilast or biologics. Apremilast was launched in Japan in March 2017, but the study period started in 2015; thus, some patients in 
the non-apremilast group were followed in 2015 and 2016. Therefore, the treatment patterns in 2015-2016 may be different from those after March 2017, possibly affecting the results. In this regard, the number of biologics used to treat psoriasis has been increasing since the 2010s, and the rate of biologic use has also tended to increase each year. The incidence rate of first biologic therapy use and the time to first biologic therapy use in the non-apremilast group, who were followed relatively early, may have been underestimated. Thus, the results of this study may be conservative.

\section{CONCLUSION}

In conclusion, this study found that patients treated with apremilast had a lower incidence of biologic use compared with non-apremilast users, suggesting that treatment with apremilast may ultimately reduce the clinical and cost burden on patients with psoriasis. Time to first biologic therapy use was also longer among patients treated with apremilast. Because biologic therapy is associated with various treatment burdens, reducing or delaying the need to use biologic therapy may be a benefit of apremilast to patients and society. Since this study was conducted using data obtained within a short period after the launch of apremilast in Japan, it is desirable to conduct further research when more data are accumulated.

\section{ACKNOWLEDGEMENTS}

The authors gratefully thank Kenji Adachi and Yoko Yoshinaga at Amgen K.K. for their helpful advice regarding this manuscript and Takamitsu Inami at Amgen K.K. for coordinating internal review processes for the publication.

Funding. This study and the journal's Rapid Service Fees were sponsored by Amgen Inc.

Medical Writing, Editorial, and Other Assistance. Editorial support was funded by Amgen Inc. and provided by Larry Radican,
PhD, MPH, of Peloton Advantage, LLC, an OPEN Health company, and Cathryn M. Carter, MS, employee of and stockholder in Amgen Inc.

Authorship. All named authors meet the International Committee of Medical Journal Editors (ICMJE) criteria for authorship for this article, take responsibility for the integrity of the work as a whole, and have given their approval for this version to be published.

Author Contributions. Conceptualization, methodology, and design: Masayuki Tanaka, Yasushi Ozeki, Fujio Matsuyama, and Tatsunori Murata; Data acquisition, data analysis, and interpretation: Masayuki Tanaka, Yasushi Ozeki, Fujio Matsuyama, Tatsunori Murata, Shinichi Imafuku, and Taichi Nakamura; Writing-original draft preparation: Fujio Matsuyama and Tatsunori Murata; Writing-review and editing: Masayuki Tanaka, Yasushi Ozeki, Fujio Matsuyama, Tatsunori Murata, Shinichi Imafuku, and Taichi Nakamura.

Prior Publication. Part of the data in this article was published at the 35th Annual Meeting of the Japanese Society for Psoriasis Research held online, September 12-13, 2020.

Disclosures. Masayuki Tanaka, Yasushi Ozeki, and Taichi Nakamura are employees of Amgen K.K. Fujio Matsuyama and Tatsunori Murata are employees of Crecon Medical Assessment Inc. and received consultation fees from Amgen K.K. for protocol drafting, statistical analysis, and manuscript drafting. Shinichi Imafuku has received grants and personal fees from AbbVie, Eisai, Janssen, Kyowa Kirin, LEO Pharma, Maruho, Mitsubishi Tanabe, Sun Pharma, Taiho Yakuhin, and Torii Yakuhin and personal fees from Amgen, Bristol Myers Squibb, Daiichi Sankyo, Eli Lilly, Novartis, and UCB.

Compliance with Ethics Guidelines. No institutional or ethics review was required because this analysis used only retrospective healthcare claims data and did not involve the collection, use or transmittal of individually identifiable data. 
Data Availability. The datasets generated during and/or analyzed during the current study are available from the corresponding author on reasonable request.

Open Access. This article is licensed under a Creative Commons Attribution-NonCommercial 4.0 International License, which permits any non-commercial use, sharing, adaptation, distribution and reproduction in any medium or format, as long as you give appropriate credit to the original author(s) and the source, provide a link to the Creative Commons licence, and indicate if changes were made. The images or other third party material in this article are included in the article's Creative Commons licence, unless indicated otherwise in a credit line to the material. If material is not included in the article's Creative Commons licence and your intended use is not permitted by statutory regulation or exceeds the permitted use, you will need to obtain permission directly from the copyright holder. To view a copy of this licence, visit http://creativecommons.org/licenses/by$\mathrm{nc} / 4.0 /$.

\section{REFERENCES}

1. Saeki H, Terui T, Morita A, Sano S, Imafuku S, Asahina $\mathrm{A}$, et al. Japanese guidance for use of biologics for psoriasis (the 2019 version). J Dermatol. 2020;47(3):201-22.

2. Lebwohl MG, Bachelez H, Barker J, Girolomoni G, Kavanaugh A, Langley RG, et al. Patient perspectives in the management of psoriasis: results from the population-based Multinational Assessment of Psoriasis and Psoriatic Arthritis Survey. J Am Acad Dermatol. 2014;70(5):871-81.

3. Takahashi H, Satoh K, Takagi A, Iizuka H. Economic burden of psoriatic patients in Japan: analysis from a single outpatient clinic. J Dermatol. 2017;44(9): 1024-6.

4. Papp K, Reich K, Leonardi CL, Kircik L, Chimenti S, Langley RG, et al. Apremilast, an oral phosphodiesterase 4 (PDE4) inhibitor, in patients with moderate to severe plaque psoriasis: results of a phase III, randomized, controlled trial (Efficacy and Safety Trial Evaluating the Effects of Apremilast in Psoriasis [ESTEEM 1]). J Am Acad Dermatol. 2015;73(1): $37-49$.
5. Paul C, Cather J, Gooderham M, Poulin Y, Mrowietz U, Ferrandiz C, et al. Efficacy and safety of apremilast, an oral phosphodiesterase 4 inhibitor, in patients with moderate to severe plaque psoriasis over 52 weeks: a phase III, randomized, controlled trial (ESTEEM 2). Br J Dermatol. 2015;173(6): 1387-99.

6. Ohtsuki M, Okubo Y, Komine M, Imafuku S, Day RM, Chen P, et al. Apremilast, an oral phosphodiesterase 4 inhibitor, in the treatment of Japanese patients with moderate to severe plaque psoriasis: efficacy, safety and tolerability results from a phase $2 \mathrm{~b}$ randomized controlled trial. J Dermatol. 2017;44(8):873-84.

7. Kishimoto M, Komine M, Hioki T, Kamiya K, Sugai J, Ohtsuki M. Real-world use of apremilast for patients with psoriasis in Japan. J Dermatol. 2018;45(11):1345-8.

8. Ohata C, Ohyama B, Kuwahara F, Katayama E, Nakama T. Real-world data on the efficacy and safety of apremilast in Japanese patients with plaque psoriasis. J Dermatol Treat. 2019;30(4):383-6.

9. Saruwatari H. Real-world experiences of apremilast in clinics for Japanese patients with psoriasis. J Dermatol. 2019;46(12):1166-9.

10. Kishimoto M, Komine M, Kamiya K, Sugai J, Ohtsuki M. Drug survival of apremilast in a real-world setting. J Dermatol. 2019;46(7):615-7.

11. Japanese Society for Pharmacoepidemiology, Drug Epidemiology and Database Task Force Activities. Survey of Japanese databases in Japan available for clinical/pharmaco-epidemiology. September 2019. http://www.jspe.jp/committee/020/0210/. Accessed 19 Jan 2021.

12. Tada Y, Ishii K, Kimura J, Hanada K, Kawaguchi I. Patient preference for biologic treatments of psoriasis in Japan. J Dermatol. 2019;46(6):466-77.

13. Feldman SR, Pelletier CL, Wilson KL, Mehta RK, Brouillette MA, Smith D, et al. Real-world US healthcare costs of psoriasis for biologic-naive patients initiating apremilast or biologics. J Comp Eff Res. 2019;8(1):45-54.

14. Wu JJ, Pelletier C, Ung B, Tian M. Real-world treatment patterns and healthcare costs among biologic-naive patients initiating apremilast or biologics for the treatment of psoriasis. J Med Econ. 2019;22(4):365-71.

15. Feldman SR, Zhang J, Martinez DJ, Lopez-Gonzalez L, Marchlewicz EH, Shrady G, et al. Real-world treatment patterns and healthcare costs of biologics and apremilast among patients with moderate-to- 
severe plaque psoriasis by metabolic condition status. J Dermatol Treat. 2021;32:203-11.

16. Imafuku S, Nakano A, Dakeshita H, Li J, Betts KA, Guerin A. Number needed to treat and costs per responder among biologic treatments for moderateto-severe plaque psoriasis in Japan. J Dermatol Treat. 2018;29(1):24-31.

17. Sruamsiri R, Iwasaki K, Tang W, Mahlich J. Persistence rates and medical costs of biological therapies for psoriasis treatment in Japan: a real-world data study using a claims database. BMC Dermatol. 2018;18(1):5.

18. Aljaser M, Amar L, Kircik LH. Use of apremilast in patients who are dissatisfied with stable maintenance topical therapy. J Drugs Dermatol. 2019;18(4):336-40.

19. Bagel J, Nelson E, Keegan BR. Apremilast and narrowband ultraviolet-B combination therapy for treating moderate-to-severe plaque psoriasis. J Drugs Dermatol. 2017;16(10):957-62. 